\title{
Contamination Effects and Requirements Derivation for the James Webb Space Telescope
}

\author{
Dr. Jonathan Arenberg \\ Northrop Grumman Space Technology \\ and \\ Eve Wooldridge \\ NASA/Goddard Space Flight Center
}

\section{Witth Thanks To ....}

The many people who form or support the JWST contamination control team with many hours and many calculations:

Dr. Mark Clampin, Observatory Scientist

Dr. Paul Lightsey, Observatory Optical Systems Engineer

and

From NGST: Robert Lempka, Matthew Macias, Dr. Dan McGregor, Suzanne Casement, Robert Giampaoli, Michael Hirsch

From GSFC: Mark Hasegawa, Ray LeVesque, Dr. Michael Woronowicz, Dr. Kevin Novo-Gradac, Karrie Houston, Larissa Graziani, Jack Triolo, Michael Menzel 


\section{Mission Requirements Lead to Contamination Requirements}

- What is the James Webb Space Telescope (JWST)? What is JWST's mission?

- How does contamination effect the JWST mission, its science performance?

- Sensitivity

- Stray Light

- Transmission

- Derivation of JWST Contamination Requirements

\section{What is JWST? Why is Contamination an lssue?}

- JWST is a large, passively cooled, $6.5 \mathrm{~m}$ diameter telescope operating from $\sim 0.6 \mu \mathrm{m}$ to $29 \mu \mathrm{m}$ designed to detect First Light in the Cosmic Dark Age, and to

- study the reionization initiated by the First Light,

- the assembly of galaxies,

- the birth of stars and protoplanetary systems,

- planetary systems and the origins of life.

- Passive cooling means an open architecture

- Large exposed sensitive areas

- Detection of First Light leads to the need for the large optical collection area, which must also be very sensitive

- Long wavelengths means

- telescope is cold, $30-50 \mathrm{~K}$ optics temperature

- need to be very careful about venting and cool-down profile

- Stray light is a key issue

- mirrors will require stringent particulate requirement 


\section{JWST is Huge}

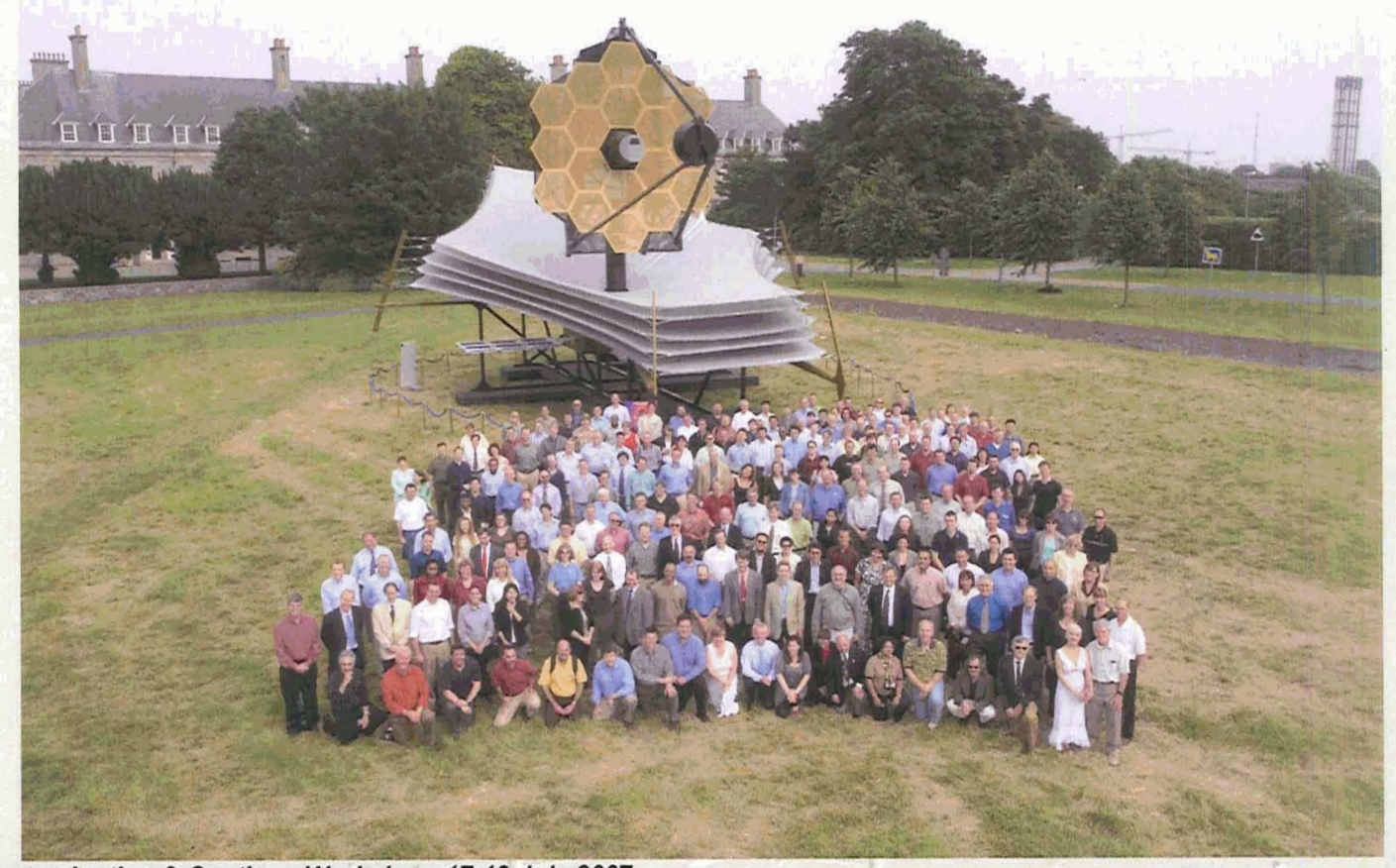

\section{Deplloyed +لJ1] V/iew}

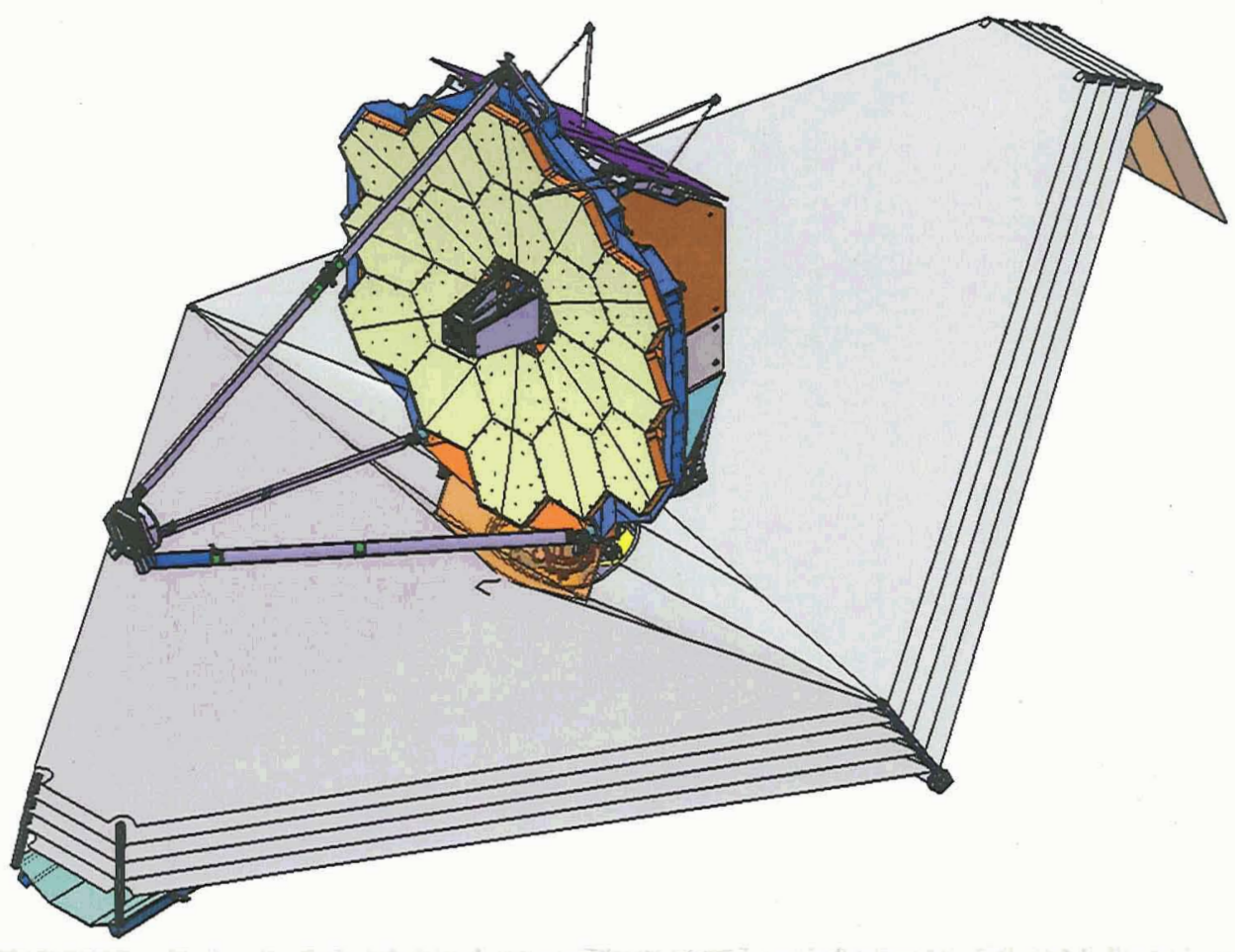




\section{Deplloyed View}

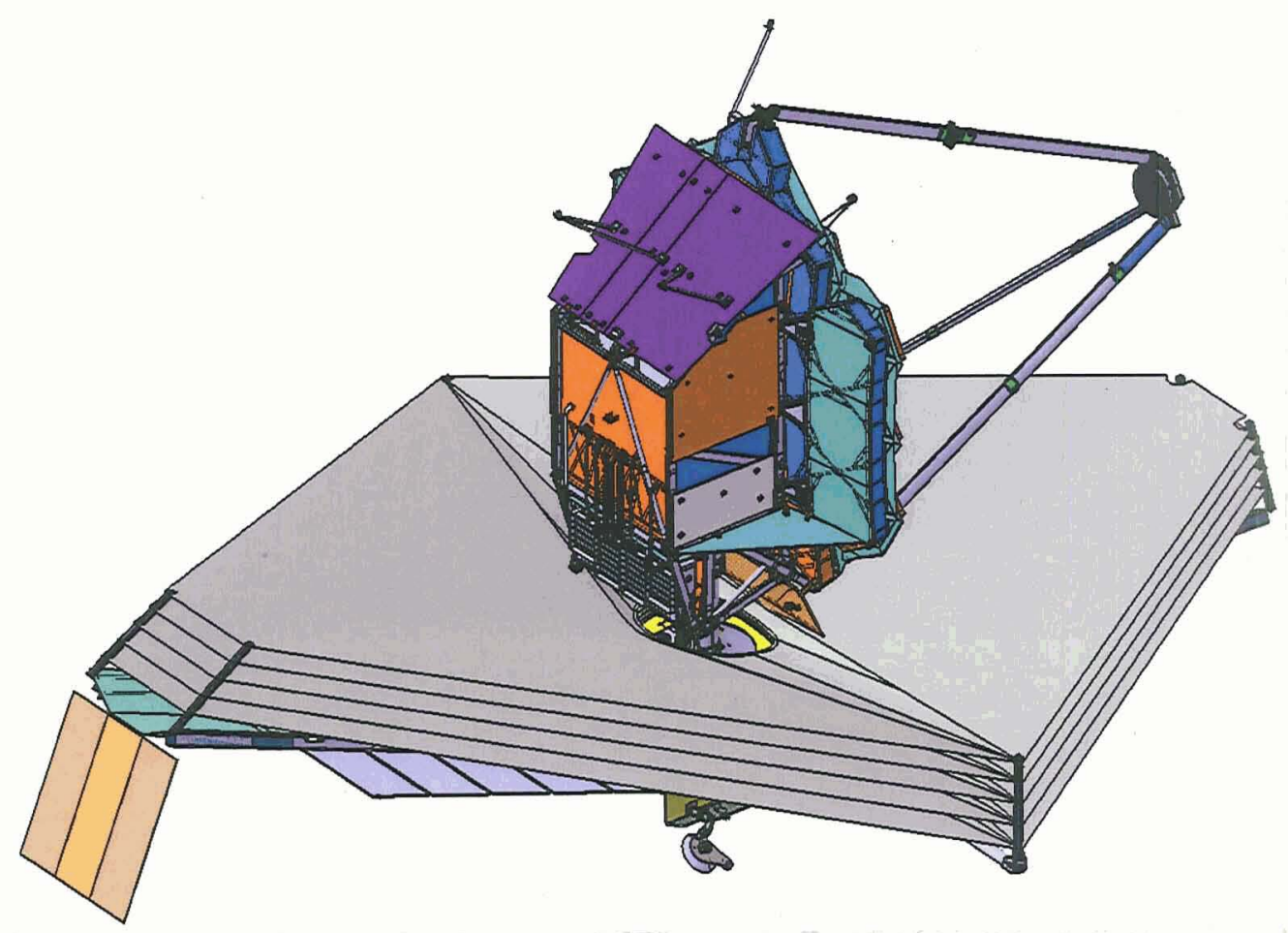

Contamination \& Coatings Workshop, 17-19 July 2007

\section{Stowedl Views of Baselline 2}
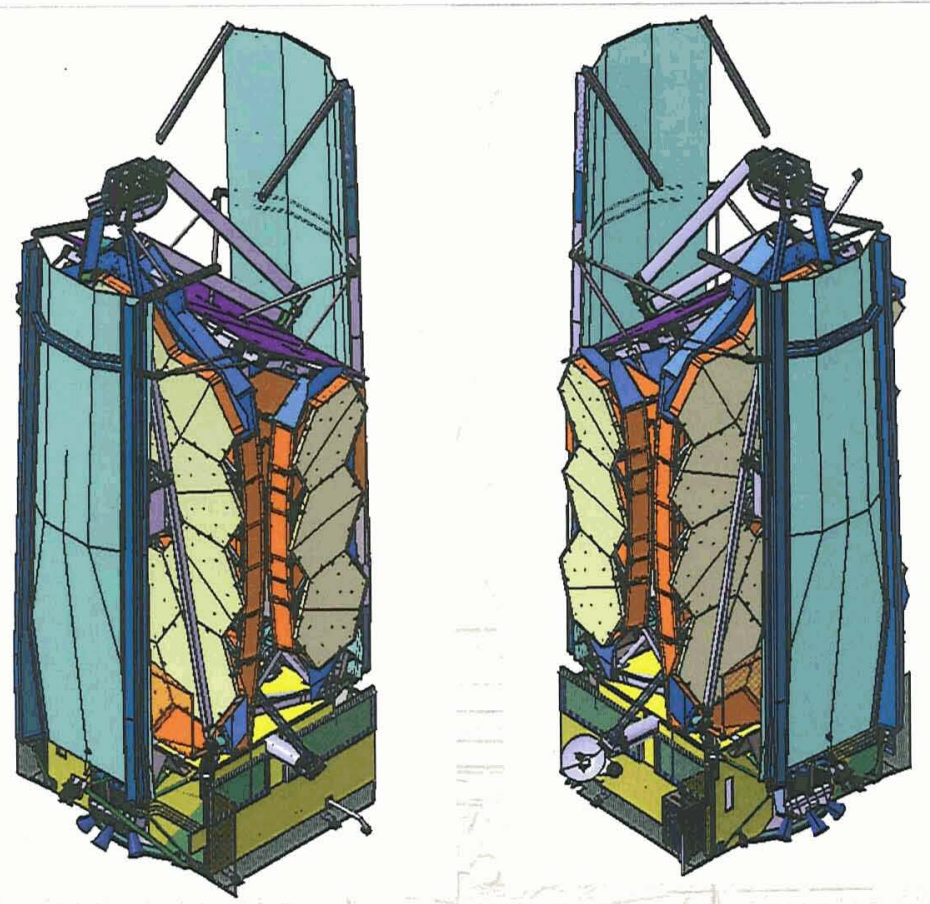


\section{JWST's Foun SGienge Themes}

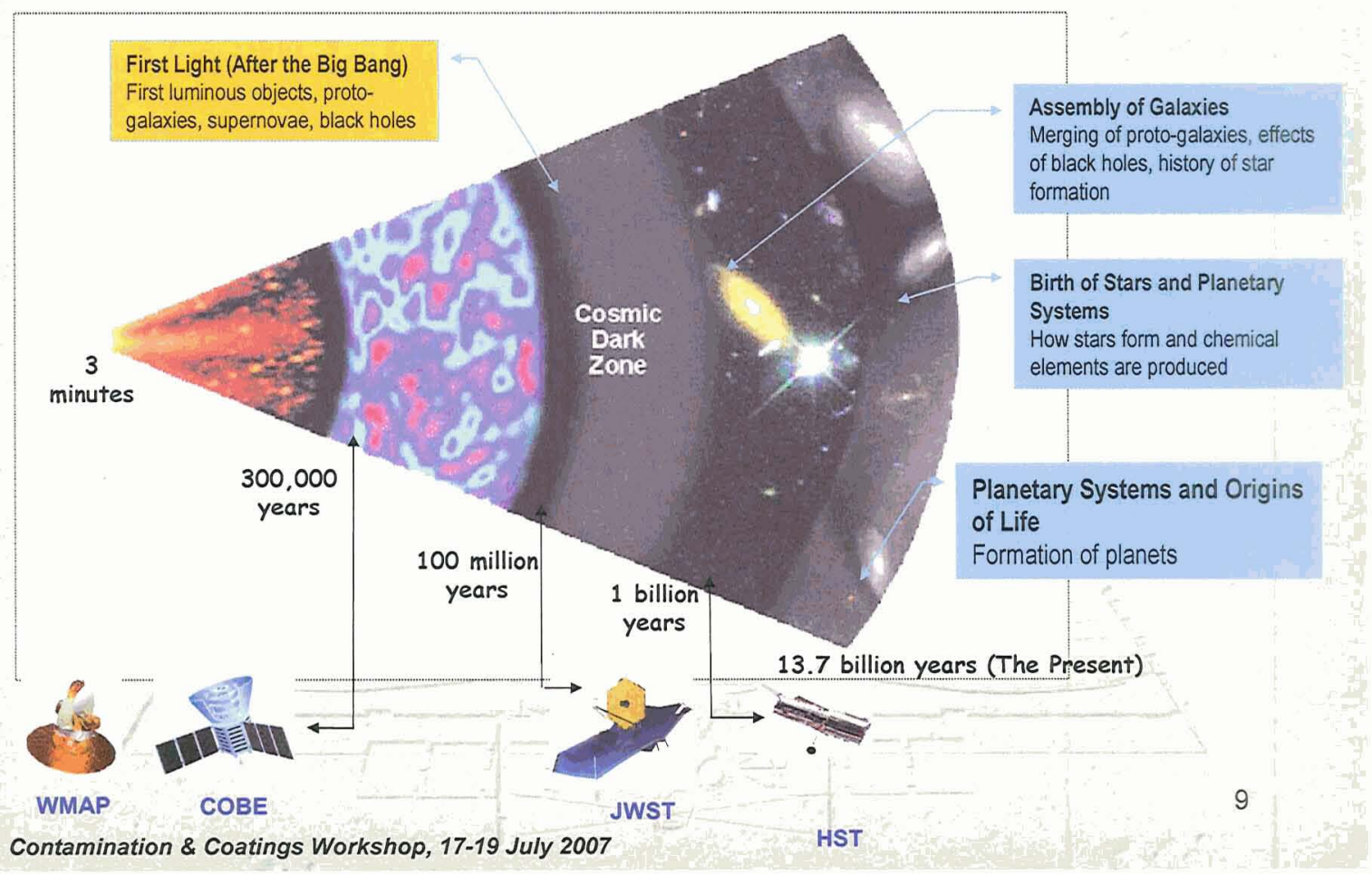

\section{Detecting "First Ligghthes}

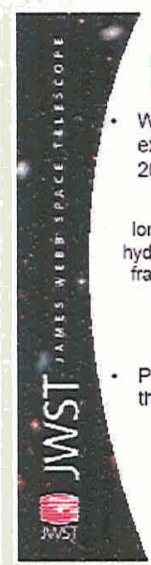

First light sources : detection

WMAP indicates that there may sources at $z=15-20 \rightarrow$ extended reionization or two relanization epochs (e.g. Cen 2003).

lonized hydrogen

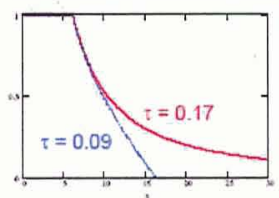

Extended reionization with $\tau$ in the range

0.09-0.17 gives first

light between 16 and 50 even if reionization is completed at $z=6$.

Probing the $L F$ to the same relative depth as that of $z=6$ from the UDF gives us a required cepth:
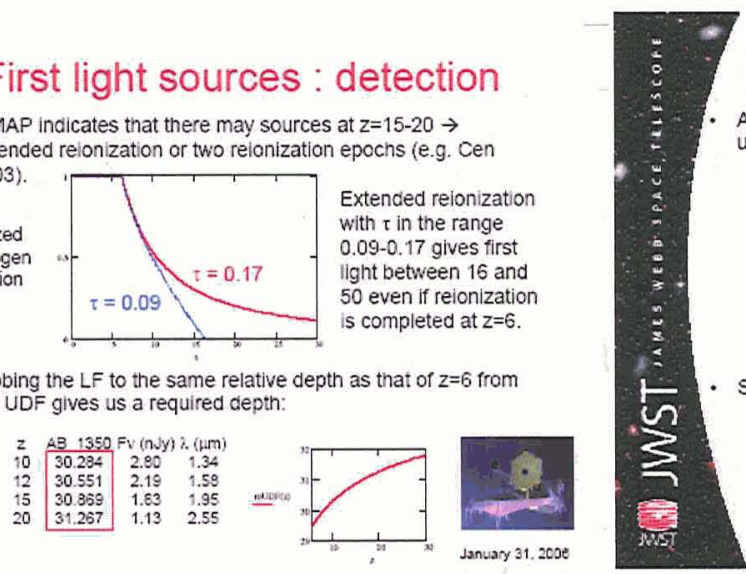

\section{First light sources : detection}

A first principle calculations gives us:

Summarizing....

\begin{tabular}{|l|c|}
\hline Method & F200W depth (nIy) \\
\hline UDF relarive depth & 1.63 for $z=15,1.13$ tor $z=20$ \\
\hline Nos-arolvas LF fiom $z=6$ & 14 for $z=13,10$ for $z=17$ \\
\hline Ab-initio & 0.2 for $z=10$ \\
\hline
\end{tabular}

From M. Stiavelli et al. JWST SWG, February'06

The time it will take to measure a $1 \mathrm{nJy}$ source - that of "First Light" at $2 \mu \mathrm{m}$ to a $\mathrm{S} / \mathrm{N}=10$ is sensitive metric of the absolute science performance of the JWST mission.

If stray light exceeds requirements and transmission does not meet requirements, the prime science mission of detecting First Light will be compromised. 


\section{How dloes contamination effect Scienge Performange?}

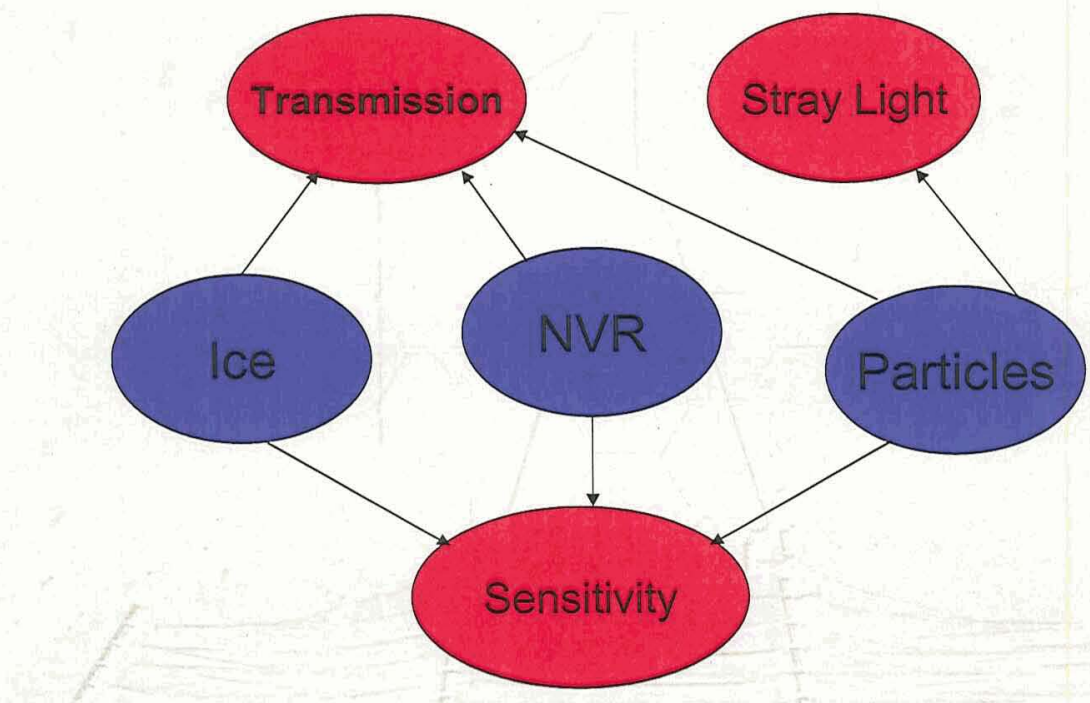

\section{JWST Mission Requilrements}

- MR-51

- Level 2 mission requirement for observatory sensitivity

- Measured in $\mathrm{W} / \mathrm{m}^{2} \mathrm{~Hz}$ for $\mathrm{SN}$ and $\mathrm{R}$ values

- Effects the integration time necessary to make an observation

- Determined by calculation for each instrument; stray light levels and transmission effect sensitivity

- MR-121

- Level 2 mission requirement for stray light/particulate requirements

- Stray light spectral radiance level measured in Mega Janskeys (MJy)

$-1 \mathrm{MJy} / \mathrm{sr}=1 \times 10-20 \mathrm{~W} / \mathrm{m}^{2-} \mathrm{Hz}-\mathrm{sr}$

- Particulate contamination increases stray light radiance

- MR-211

- Level 2 mission requirement for transmission

- Optical transmission requirement, measured in \%

- The required \% transmission accounts for transmission losses from coatings, dust, obscuration, meteoroid damage, and contamination

- Thin film interference by NVR and water/ice and Percent Area Coverage (PAC) of particulates effect transmission 


\section{The Sensititivity Requilrement (MR-51))}

The observatory system shall reach the sensitivity performance levels shown in the following table when observing a position on the celestial sphere that exhibits 1.2 times the minimum Zodiacal light background power as calculated in the NIRCam, NIRSpec, MIRI, and FGS-TF Sensitivity Calculations.

\begin{tabular}{|c|c|l|}
\hline $\begin{array}{c}\text { Wavelength } \\
(\mu \mathrm{m})\end{array}$ & Instrument/Mode & \multicolumn{1}{|c|}{ Sensitivity } \\
\hline 1.15 & NIRCam/WFS & $1.10 \times \mathrm{E}-31 \mathrm{Wm}^{-2} \mathrm{~Hz}^{-1} \mathrm{SN}=10$ in 10.6 s or less $\& \mathrm{R}=4$ bandwidth \\
\hline 2 & NIRCam & $1.14 \times \mathrm{E}-34 \mathrm{Wm}^{-2} \mathrm{~Hz}^{-1} \mathrm{SN}=10$ in 10,000 s or less $\& \mathrm{R}=4$ bandwidth \\
\hline 3.5 & FGS-TF & $1.26 \times \mathrm{E}-33 \mathrm{Wm}^{-2} \mathrm{~Hz}{ }^{-1} \mathrm{SN}=10$ in 10,000 s or less $\& \mathrm{R}=100$ bandwidth \\
\hline 3.0 & NIRSpec/Low Res & $1.32 \times \mathrm{E}-33 \mathrm{Wm}^{-2} \mathrm{~Hz}^{-1} \mathrm{SN}=10$ in 10,000 s or less $\& \mathrm{R}=100$ bandwidth \\
\hline 2.0 & NIRSpec/Med Res & $5.2 \times \mathrm{E}-22 \mathrm{Wm}^{-2} \mathrm{~Hz}^{-1} \quad \mathrm{SN}=10$ in 10,000 s or less \\
\hline 10 & MIRI/Broad-Band & $7.0 \times \mathrm{E}-33 \mathrm{Wm}^{-2} \mathrm{~Hz}^{-1} \mathrm{SN}=10$ in 10,000 s or less $\& \mathrm{R}=5$ bandwidth \\
\hline 21 & MIRI/Broad-Band & $8.7 \times \mathrm{E}-32 \mathrm{Wm}^{-2} \mathrm{~Hz}^{-1} \mathrm{SN}=10$ in 10,000 s or less \& $\mathrm{R}=4.2$ bandwidth \\
\hline 9.2 & MIRI/Spectrometer & $1.0 \times \mathrm{E}-20 \mathrm{Wm}^{-2} \mathrm{~Hz}^{-1} \mathrm{SN}=10$ in 10,000 s or less \& $\mathrm{R}=2400$ bandwidth \\
\hline 22.5 & MIRI/Spectrometer & $5.6 \times \mathrm{E}-20 \mathrm{Wm}^{-2} \mathrm{~Hz}^{-1} \mathrm{SN}=10$ in 10,000 s or less \& $\mathrm{R}=2400$ bandwidth \\
\hline
\end{tabular}

1 JWST Mission Requirements Document, JWST-RQMT-00634, Revision N, 30 August 2006

\section{History of the changing sensitivitty of JW/ST att 2 microrn = malinlly dulue to the increased alllocatijon for stiraly/ lightht}

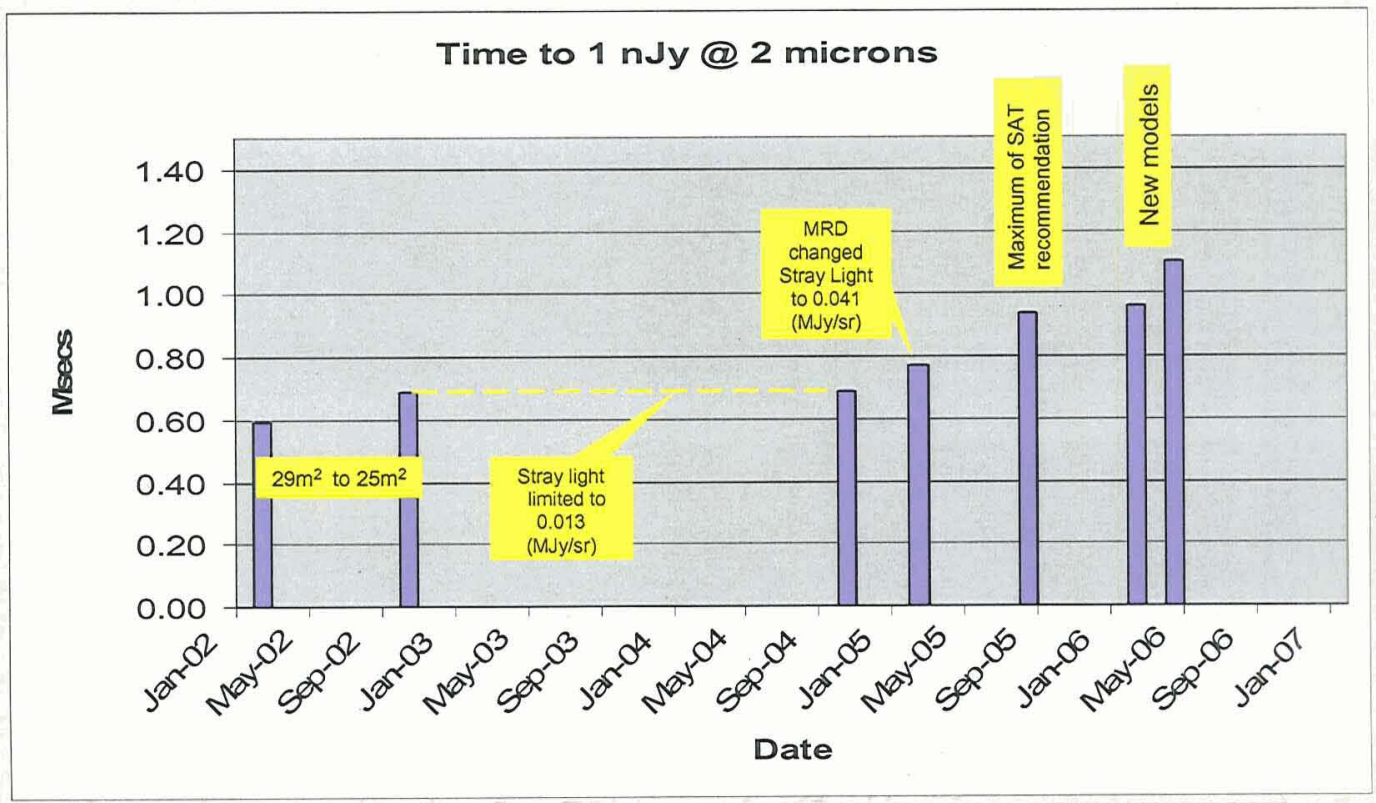




\section{Stray Light is a signifitiant sourre of JWST sensitivity loss for IIR Tellescopes}

- Violation of the stray light requirements has been one of the greatest threats to meeting JWST's sensitivity requirements.

- Contributions to stray light

- Zodi background (infield)

- Zodi background (out field)

- Galactic sky

- Earth \& moon

- Nearby bright field stars

- Rogue path

\section{Signall-to=Noise Ratio Depends on Stray/ Light}

$$
S N R=\frac{S}{\sqrt{S+Z+S L_{\text {OTE }}+S L_{\text {ISIM }}+D+R d}}
$$

- $\mathrm{S}=$ target signal

- Z = zodiacal background

- $\mathrm{SL}_{\mathrm{OTE}}=$ stray light background signal from OTE/SC

- $S L_{\text {ISIM }}=$ stray light from background signal from ISIM

- $D=$ total integrated dark current

- $\mathrm{R}=$ read noise (variance) 


\section{The JWST Stray Light Requilirement "}

When observing a position on the celestial sphere that exhibits 1.2 times the minimum Zodiacal light background radiance, the stray light incident into an instrument acceptance cone at the instrument pickoff mirror shall be less than an equivalent background in the field of view having a spectral radiance at the wavelengths and exclusion angles given in the table below.

\begin{tabular}{|c|c|c|c|c|c|}
\hline \multicolumn{2}{|c|}{$\begin{array}{c}\text { Radiance } \\
\left(1 \times 10^{-20} \mathrm{~W} /\left(\mathrm{m}^{2}-\mathrm{Hz}-\mathrm{sr}\right)\right. \\
(\mathrm{MJy} / \mathrm{sr})\end{array}$} & \multicolumn{4}{|c|}{ Exclusion Angle (degrees) } \\
\cline { 2 - 6 } & & 1.0 & 0.50 & 0.25 & 0.10 \\
\hline \multirow{2}{*}{$\begin{array}{c}\text { Wavelength } \\
(\mu \mathrm{m})\end{array}$} & 2.0 & 0.091 & 0.044 & 0.061 & 0.598 \\
\cline { 2 - 6 } & 3.0 & 0.032 & 0.035 & 0.042 & 0.326 \\
\hline
\end{tabular}

1 JWST Mission Requirements Document, JWST-RQMT-00634, Revision N, 30 August 2006

\section{Stray Lightit as an Fungtion of Clleanliness Level! (MMarrem 2006)}

\begin{tabular}{|c|c|c|c|c|c|c|c|}
\hline $\begin{array}{c}\text { Wavelength } \\
(\mu \mathrm{m})\end{array}$ & $\begin{array}{c}\text { In-field Zodi } \\
(\mathrm{MJy} / \mathrm{sr})\end{array}$ & $\begin{array}{c}\text { Requirement } \\
(\mathrm{MJy} / \mathrm{sr})\end{array}$ & $\begin{array}{c}\text { Galactic Sky } \\
(\mathrm{MJy} / \mathrm{sr})\end{array}$ & $\begin{array}{c}\text { Zodi Sky } \\
(\mathrm{MJy} / \mathrm{sr})\end{array}$ & $\begin{array}{c}\text { Earth Shine } \\
(\mathrm{MJy} / \mathrm{sr})\end{array}$ & $\begin{array}{c}\text { Moon Shine } \\
\text { (MJy/sr) }\end{array}$ & $\begin{array}{c}\text { Out-field Total } \\
\text { (MJy/sr) }\end{array}$ \\
\hline 1 & 0.138 & $\mathrm{na}$ & 0.039 & 0.029 & 0.096 & 0.017 & 0.181 \\
\hline 2 & 0.094 & 0.091 & 0.037 & 0.020 & 0.054 & 0.009 & 0.120 \\
\hline 3 & 0.081 & 0.032 & 0.022 & 0.008 & 0.033 & 0.012 & 0.075 \\
\hline 5 & 0.46 & na & 0.019 & 0.001 & 0.454 & 0.192 & 0.666 \\
\hline
\end{tabular}

Level 630

\begin{tabular}{|c|c|c|c|c|c|c|c|}
\hline $\begin{array}{c}\text { Wavelength } \\
(\mu \mathrm{m})\end{array}$ & $\begin{array}{c}\text { In-field Zodi } \\
(\mathrm{MJy} / \mathrm{sr})\end{array}$ & $\begin{array}{c}\text { Requirement } \\
(\mathrm{MJy} / \mathrm{sr})\end{array}$ & $\begin{array}{c}\text { Galactic Sky } \\
(\mathrm{MJy} / \mathrm{sr})\end{array}$ & $\begin{array}{c}\text { Zodi Sky } \\
(\mathrm{MJy} / \mathrm{sr})\end{array}$ & $\begin{array}{c}\text { Earth Shine } \\
(\mathrm{MJy} / \mathrm{sr})\end{array}$ & $\begin{array}{c}\text { Moon Shine } \\
(\mathrm{MJy} / \mathrm{sr})\end{array}$ & $\begin{array}{c}\text { Out-field Total } \\
(\mathrm{MJy} / \mathrm{sr})\end{array}$ \\
\hline 1 & 0.138 & $\mathrm{na}$ & 0.045 & 0.033 & 0.115 & 0.020 & 0.213 \\
\hline 2 & 0.094 & 0.091 & 0.046 & 0.023 & 0.067 & 0.012 & 0.148 \\
\hline 3 & 0.081 & 0.032 & 0.026 & 0.009 & 0.036 & 0.014 & 0.085 \\
\hline 5 & 0.46 & $\mathrm{na}$ & 0.022 & 0.001 & 0.480 & 0.210 & 0.713 \\
\hline
\end{tabular}

Level 720

\begin{tabular}{|c|c|c|c|c|c|c|c|}
\hline $\begin{array}{c}\text { Wavelength } \\
(\mu \mathrm{m})\end{array}$ & $\begin{array}{c}\text { In-field Zodi } \\
(\mathrm{MJy} / \mathrm{sr})\end{array}$ & $\begin{array}{c}\text { Requirement } \\
(\mathrm{MJy} / \mathrm{sr})\end{array}$ & $\begin{array}{c}\text { Galactic Sky } \\
(\mathrm{MJy} / \mathrm{sr})\end{array}$ & $\begin{array}{c}\text { Zodi Sky } \\
(\mathrm{MJy} / \mathrm{sr})\end{array}$ & $\begin{array}{c}\text { Earth Shine } \\
(\mathrm{MJy} / \mathrm{sr})\end{array}$ & $\begin{array}{c}\text { Moon Shine } \\
(\mathrm{MJy} / \mathrm{sr})\end{array}$ & $\begin{array}{c}\text { Out-field Total } \\
(\mathrm{MJy} / \mathrm{sr})\end{array}$ \\
\hline 1 & 0.138 & na & 0.062 & 0.041 & 0.156 & 0.028 & 0.287 \\
\hline 2 & 0.094 & 0.091 & 0.062 & 0.029 & 0.091 & 0.016 & 0.198 \\
\hline 3 & 0.081 & 0.032 & 0.034 & 0.011 & 0.047 & 0.018 & 0.110 \\
\hline 5 & 0.46 & na & 0.027 & 0.001 & 0.533 & 0.244 & 0.805 \\
\hline
\end{tabular}




\section{The Particullate Requilirement}

- Further stray light analyses were performed, and from the numbers the senstivity calculated.

- In April 2006, the cleanliness requirement was set at Level 630 (or 1\% PAC) for the Primary Mirror (PM) and Secondary Mirror (SM), Level 550 (or $0.5 \%$ PAC) for the Tertiary Mirror (TM) and the Fine Steering Mirror (FSM)

- Further stray light analyses have been performed, as design improvements were made

\begin{tabular}{|c|c|c|c|c|c|c|c|c|c|}
\hline \multicolumn{10}{|c|}{ Stray Light as of March 2007 - PM \& SM at Level 630, TM and FSM at Level 550} \\
\hline \multicolumn{10}{|l|}{ 127odi } \\
\hline $\begin{array}{c}\text { Wavelengh } \\
(\mathrm{H}-\mathrm{m})\end{array}$ & $\begin{array}{c}\text { Infiedzod } \\
\text { (MVyss) }\end{array}$ & $\begin{array}{l}\text { Requiement } \\
\text { (Myss) }\end{array}$ & $\begin{array}{l}\text { Gelacic Shy } \\
\text { (MUysi) }\end{array}$ & $\begin{array}{l}\text { Zodsty } \\
\text { (MUSsr) }\end{array}$ & $\begin{array}{l}\text { EathStire } \\
\text { (MUsi) }\end{array}$ & $\begin{array}{c}\text { Moonstine } \\
\text { (MUysi) }\end{array}$ & $\begin{array}{c}m_{\infty}=+1 @ 1^{\circ} \\
(M V y s s)\end{array}$ & $\begin{array}{l}\text { Otfiedd Total } \\
\text { (Mlysin) }\end{array}$ & $\begin{array}{c}a t / / n \\
(\%)\end{array}$ \\
\hline 1 & 0.133 & $\mathrm{Ba}$ & 0.099 & 0.028 & 0.009 & 0.003 & 0.004 & 0112 & $84.1 \%$ \\
\hline 2 & 0.091 & 0.091 & 0.061 & 0.018 & 0.004 & 0.001 & 0.004 & 0.038 & $968 \%$ \\
\hline 3 & 0.083 & 0.032 & 0.047 & 0.011 & 0.005 & 0.003 & 0.004 & 0.070 & $843 \%$ \\
\hline 3.5 & 0.078 & $\overrightarrow{\Gamma a}$ & 0.040 & 0.018 & 0.008 & 0.005 & 0.004 & $\overline{0.074}$ & $94.9 \%$ \\
\hline 5 & 0.446 & $\mathrm{na}$ & 0.020 & 0.101 & 0274 & 0.003 & 0.004 & 0.471 & $1056 \%$ \\
\hline
\end{tabular}

\section{Most Regent Revision}

- Prediction of particulate level at time of launch indicated that to meet 1\% PAC on the Primary Mirror, aggressive mirror covering techniques would be required - very undesirable to optics group

- The same analysis predicted that $1.4 \%$ EOL could be met without extreme protective measures

- Systems engineering evaluated relaxing the requirement to $1.5 \%$ PAC on the Primary Mirror, and tightening the requirement to $0.5 \%$ PAC on the Secondary Mirror

- The PM is integrated cup-up, is very large and is segmented, therefore it is much harder to maintain clean

- This change would minimize risk and cost 


\section{Anallyses Perfiormed on 3 Cases}

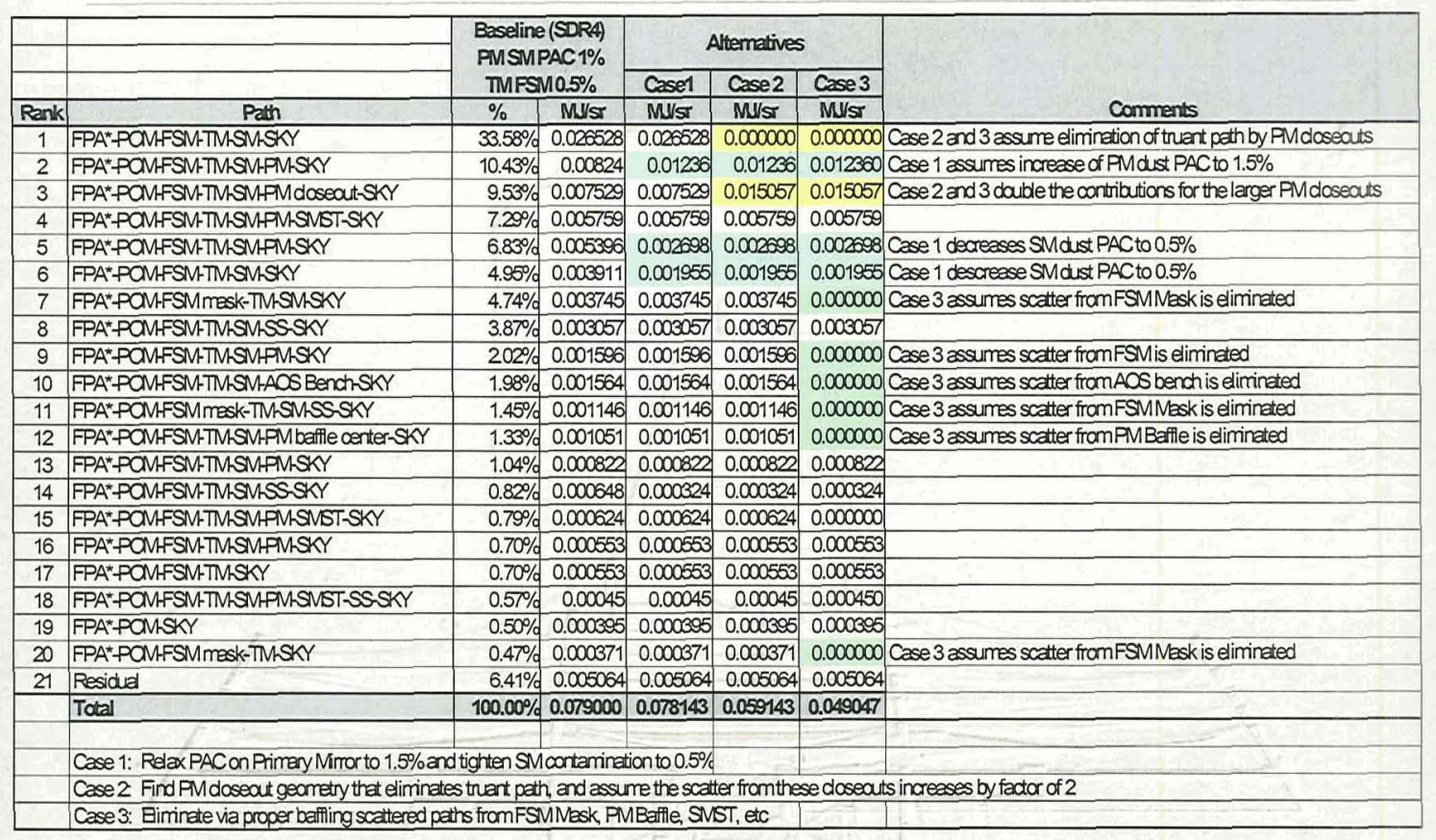

\section{The Current Particullate Requilrement}

- Improvements to design (i.e. closeouts to stray light paths) and the combination of tightening SM requirement while relaxing PM requirement led to the following, most current requirements:

$\begin{array}{ll}\text { Primary Mirror } & 1.5 \% \text { \%AC } \\ \text { Secondary Mirror } & 0.5 \% \text { \%AC } \\ \text { Fine Steering Mirror } & 0.5 \% \text { PAC } \\ \text { Tertiary Mirror } & 0.5 \% \text { PAC }\end{array}$

- Systems engineering put conditions on these changes:

- Stray Light and Transmission levels remain at their current required levels (no relaxation permitted for easing particulate requirement)

- Design features to improve stray light performance must continue to be pursued

- The analyses used to accept the change is correct and does not change significantly in the next round of analyses

- No degradation of the OTE transmission error budget is incurred because of this change 


\section{The Transmission Budget}

\section{Equally critical to Observatory Sensitivity ...}

\section{The Transmission Requirement (MR-2111)}

Accounting for all effects on mirror transmission including: coatings, dust, obscuration, meteoroid damage, and contamination, the End of Life (EOL) optical spectral transmission of the OTE shall be greater than the values shown in the following table for wavelengths between 0.8 micrometers and 2.0 micrometers, and greater than $88 \%$ for wavelengths from 2.0 micrometers to 27 micrometers, with transmission out to 29 micrometers as a goal.

\begin{tabular}{|c|c|}
\hline $\begin{array}{c}\text { Wavelength } \\
(\mu \mathrm{m})\end{array}$ & $\begin{array}{c}\text { Transmission } \\
T(\%)\end{array}$ \\
\hline 0.8 & 61.5 \\
\hline 1.5 & 82.0 \\
\hline 2.0 & 88.0 \\
\hline$>2.0$ & 88 \\
\hline
\end{tabular}

1 JWST Mission Requirements Document, JWST-RQMT-00634, Revision N, 30 August 2006 


\section{Signal Level is Dependent on Transmission}

$$
\begin{aligned}
& S=F_{v} \cdot \Delta v \cdot \tau_{\text {ote }} \cdot A \cdot \tau_{\text {instr }} \cdot Q E \cdot t_{\exp } /(h \cdot v) \\
& S=F_{\lambda} \cdot \Delta \lambda \cdot \tau_{\text {ote }} \cdot A \cdot \tau_{\text {instr }} \cdot Q E \cdot t_{\exp } \cdot \lambda /(h \cdot c)
\end{aligned}
$$

-Signal calculation given for either frequency or wavelength spectral irradiances

$-F_{v}=$ spectral irradiance (per frequency)

$-F_{\lambda}=$ spectral irradiance (per wavelength)

$-\Delta v=$ spectral bandwidth (frequency)

$-\Delta \lambda=$ spectral bandwidth (wavelength)

- texp $=$ total integration time $(100,000$ seconds $)$

$-A=$ collecting aperture area

$-\tau_{\text {OTE }}=$ transmittance of the OTE optics

$-\tau_{\text {instr }}=$ transmittance of the instrument

- $Q E$ = quantum efficiency of detector

$-v=$ frequency

$-\lambda=$ wavelength

$-h=$ Planck's constant

$-c=$ speed of light

\section{NVR and W/atter//ge Signifificant Sourre of Transmission LOSS for JWST}




\section{IGe Primer: llee Absorbs Light}
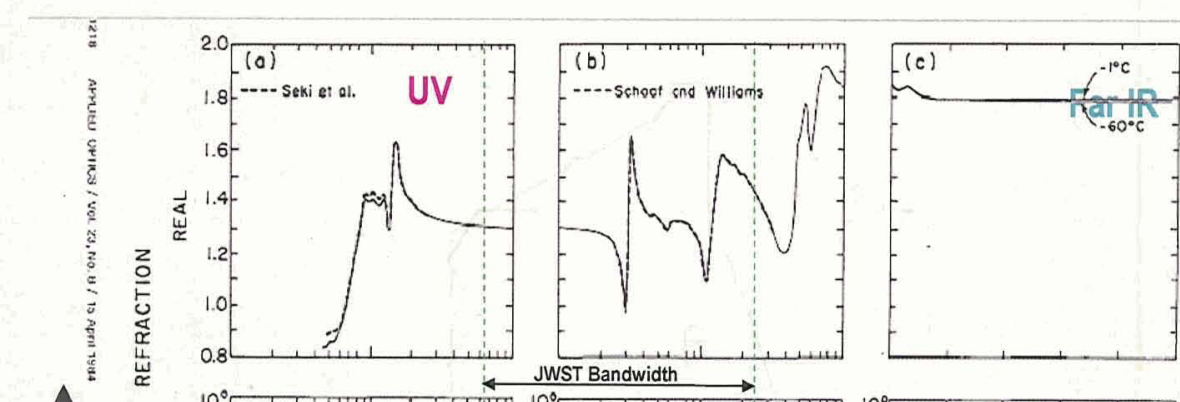

$\uparrow$

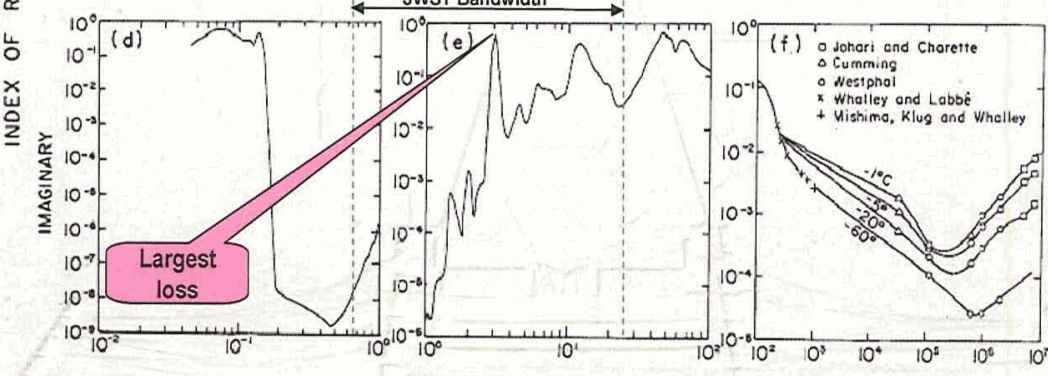

WAVELENGTH $(\mu \mathrm{m})$

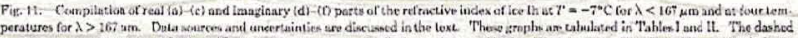

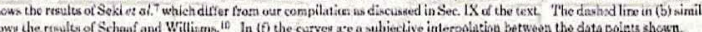

Integrated Tellescope Transmission Callewlation

- Calculate the transmission for each mirror

- Reflectance using a contamination and ice modified stack

- Angle of incidence effects

- PAC for each mirror

- Both polarizations 


\section{Beer is Not an Goodl Idlea ((At least for this a.ppliication)}

- Beer's Law ignores almost all of the physics going on

- Polarization

- Multiple reflections

- Protective layer

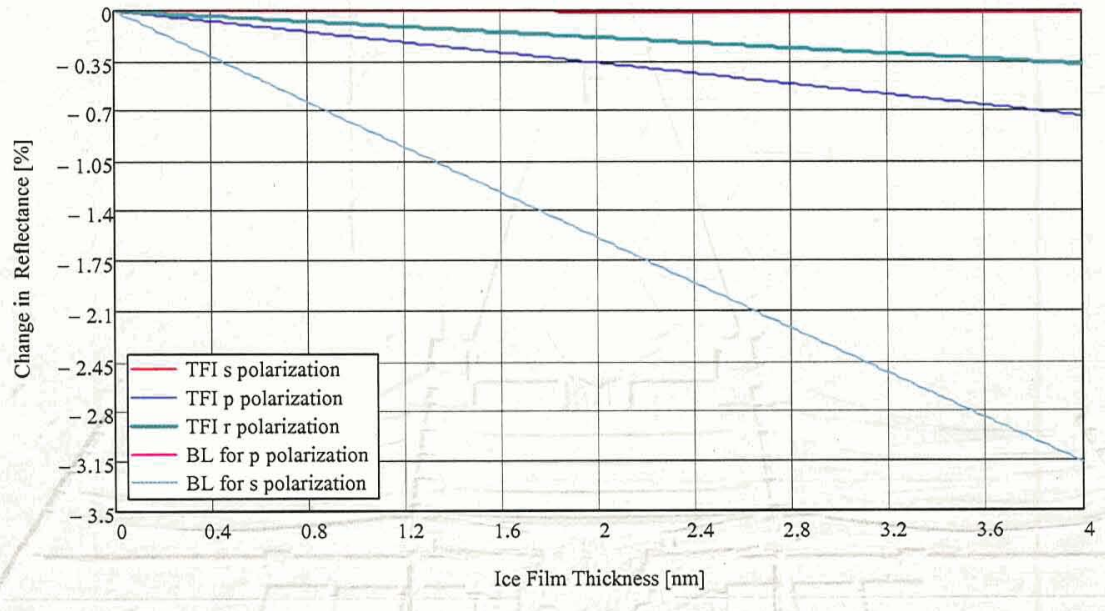

\section{Thilin Fillm Calleullations}

- The mirror (clean or contaminated) is treated as a system of films on a gold substrate

- The reflectance depends on

- Optical properties of the gold, SiOx and the contamination

- Complex index of refraction

- Gold and SiOx (SiO2) from Palik

- Ice from Warren

- NVR-"literature"

- Angle of incidence, polarization and wavelength of the incident light

- Have calculation of mirrors (thin film analysis) that includes all of the above effects

- Mathcad file

- Used to calculate reflectance of mirrors at various $\mathrm{AOI}$, ice and NVR films

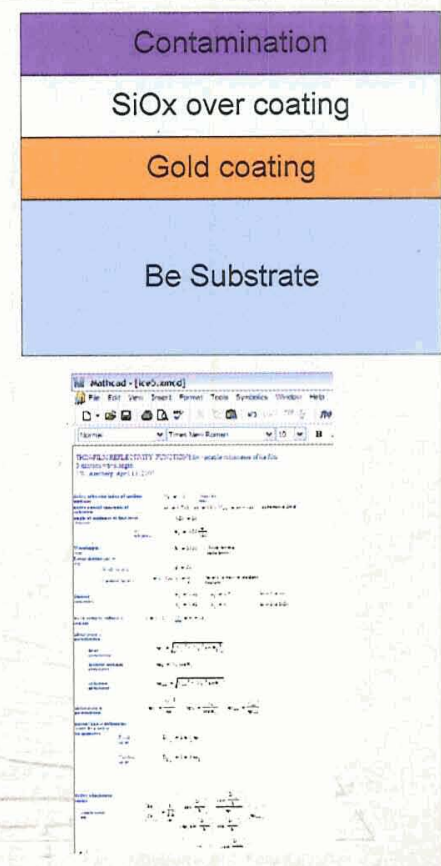




\section{NVR Impact is Hard to Assess}

- Since the indices of NVR is specific to the species and the species are unknown, so are the relevant $\mathrm{n}$ and $\mathrm{k}$ values

- Inserted $\mathrm{n}$ and $\mathrm{k}$ for an NVR layer in the TFI calculation and determined the thickness to lose $1 \%$ reflectance at normal incidence

- From the literature, we can assume and likely species have an $\mathrm{n}$ and $\mathrm{k}$ in the green circle

- $8 \mathrm{~nm}$ thickness for $1 \%$ loss

- Assume 1.2 g/cc density

$-1 \mathrm{mg} / \mathrm{cm} 2 \rightarrow$ Level A

1\% Loss Contours@i=2...m

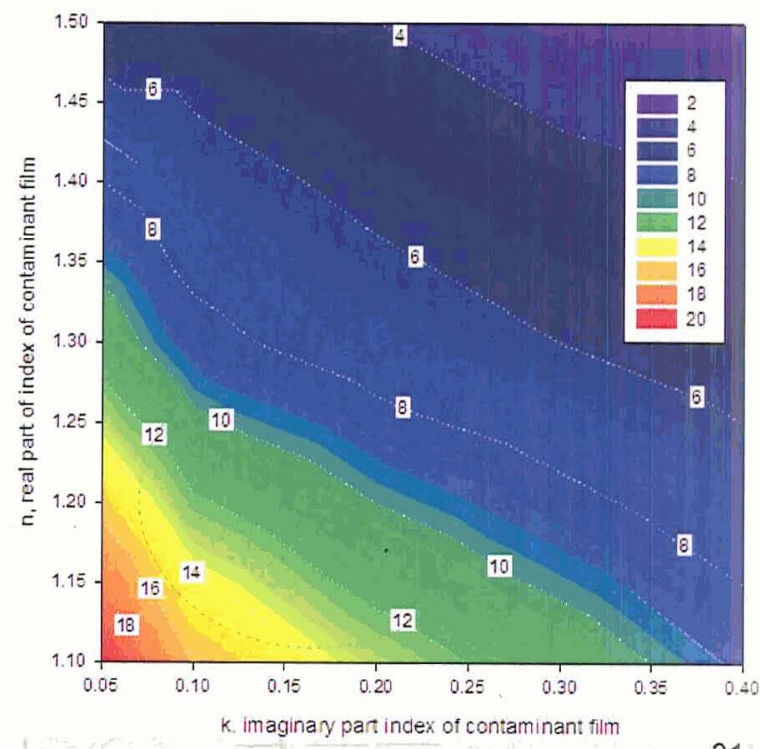

Contamination \& Coatings Workshop, 17-19 July 2007

\section{Refilection at Vacuum Material Interface}

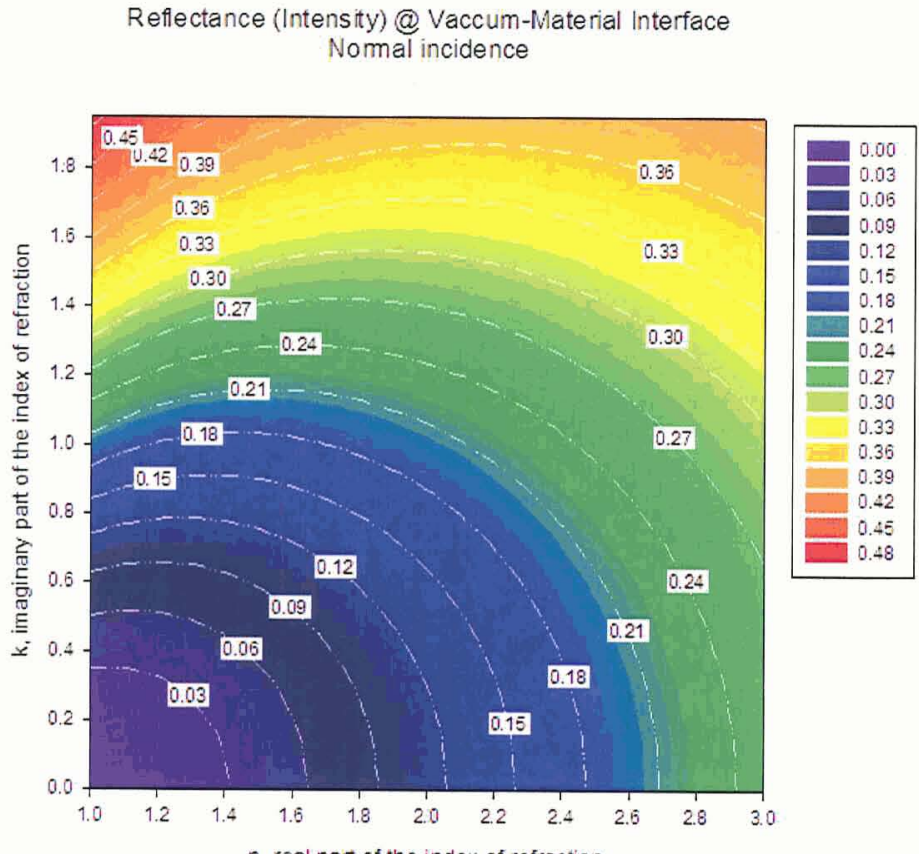

$n$ real part of the index of refraction 


\section{Talble of Contamination Requirements in the CCP}

Table I. EOI Clesnliness Recuirements for OTE Mirrors

\begin{tabular}{|c|c|c|c|c|}
\hline Mirzor & $\begin{array}{c}\text { Farticulate } \\
\text { (Fercent Area Coverage, PAC) }\end{array}$ & $\begin{array}{c}\text { Moleculas: } \\
\text { (angstroms, } A)\end{array}$ & 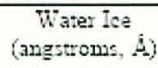 & $\begin{array}{l}\text { Amonphous Carbon } \\
\text { (angetroms, } \hat{A})\end{array}$ \\
\hline $\mathrm{PM}^{27}$ & $1.0(\mathrm{TBR})$ & $200(\mathrm{TBR})$ & 200 (TBR) & 50 (TBR) \\
\hline SMA & $1.0(\mathrm{TBR})$ & $200(\mathrm{TBR})^{6]}$ & 200 (TBR) & 50 (TBR) \\
\hline TMA & 0.5 & $200(\mathrm{TBR})^{6)}$ & $100(\mathrm{TBR})$ & $N / A$ \\
\hline FSM & 0.5 & $200(\mathrm{TBR})^{\circ}$ & $0(\mathrm{TBR})$ & $\mathrm{NA}$ \\
\hline
\end{tabular}

(1) 200 Angstoms is equivalent to Level B pe: IEST-STD-CC1246D assuming a uniform molecular film of unit density. For conversions, refer to Appendiv D.

(2) The vilus apecified for the OTE Primary Mirror are reprecentative of an areage of the 1 S FMSA.

PM now $1.5 \%$, SM now $0.5 \%$

\section{EOL Transmission Budget}

2007.05.01 Budget (CCP Table 1 compatible)

\begin{tabular}{|c|c|c|c|c|c|c|c|}
\hline \multirow{2}{*}{ mirror } & \multirow{2}{*}{ reflectivity } & \multicolumn{5}{|c|}{$\lambda(u m)$} & \multirow{8}{*}{$\begin{array}{l}\text { spec * } \\
1.0 \% \text { PAC } \\
20 \mathrm{~nm} \\
20 \mathrm{~nm} \\
5 \mathrm{~nm} \\
0.003 \% \text { est. }\end{array}$} \\
\hline & & 0.8 & 1.0 & 1.5 & 2.0 & 3.095 & \\
\hline \multirow{6}{*}{ PM } & coating & 0.960 & 0.970 & 0.975 & 0.980 & 0.986 & \\
\hline & particulate & 0.990 & 0.990 & 0.990 & 0.990 & 0.990 & \\
\hline & nur & 0.991 & 0.996 & 0.998 & 0.999 & 0.995 & \\
\hline & ice & 0.995 & 0.998 & 0.999 & 0.999 & 0.990 & \\
\hline & carbon & 0.997 & 0.998 & 0.999 & 1.000 & 1.000 & \\
\hline & $\mu$-meteor & 1.000 & 1.000 & 1.000 & 1.000 & 1.000 & \\
\hline \multirow{6}{*}{$S M$} & coating & 0.960 & 0.970 & 0.975 & 0.980 & 0.986 & \multirow{6}{*}{$\begin{array}{l}1.0 \% \text { PAC } \\
20 \mathrm{~nm} \\
20 \mathrm{~nm} \\
5 \mathrm{~nm} \\
0.003 \% \text { est. }\end{array}$} \\
\hline & particulate & 0.990 & 0.990 & 0.990 & 0.990 & 0.990 & \\
\hline & nvr & 0.991 & 0.996 & 0.998 & 0.999 & 0.995 & \\
\hline & ice & 0.995 & 0.998 & 0.999 & 0.999 & 0.990 & \\
\hline & carbon & 0.997 & 0.998 & 0.999 & 1.000 & 1.000 & \\
\hline & $\mu$-meteor & 1.000 & 1.000 & 1.000 & 1.000 & 1.000 & \\
\hline \multirow{6}{*}{ TM } & coating & 0.960 & 0.970 & 0.975 & 0.980 & 0.986 & \multirow{14}{*}{$\begin{array}{l}0.5 \% \text { PAC } \\
20 \mathrm{~nm} \\
10 \mathrm{~nm} \\
\text { none } \\
0.003 \% \text { est. } \\
\text { spec }{ }^{*} \\
0.5 \% \text { PAC } \\
20 \text { nm } \\
\text { none } \\
\text { none } \\
0.003 \% \text { est. }\end{array}$} \\
\hline & particulate & 0.995 & 0.995 & 0.995 & 0.995 & 0.995 & \\
\hline & $n \mathrm{Wr}$ & 0.991 & 0.996 & 0.998 & 0.999 & 0.995 & \\
\hline & ice & 0.998 & 0.999 & 0.999 & 1.000 & 0.996 & \\
\hline & carbon & 1.000 & 1.000 & 1.000 & 1.000 & 1.000 & \\
\hline & $\mu$-meteor & 1.000 & 1.000 & 1.000 & 1.000 & 1.000 & \\
\hline \multirow{6}{*}{ FSM } & coating & 0.960 & 0.970 & 0.975 & 0.980 & 0.986 & \\
\hline & particulate & 0.995 & 0.995 & 0.995 & 0.995 & 0.995 & \\
\hline & nur & 0.991 & 0.996 & 0.998 & 0.999 & 0.995 & \\
\hline & ice & 1.000 & 1.000 & 1.000 & 1.000 & 1.000 & \\
\hline & carbon & 1.000 & 1.000 & 1.000 & 1.000 & 1.000 & \\
\hline & $\mu$-metear & 1.000 & 1.000 & 1.000 & 1.000 & 1.000 & \\
\hline \multicolumn{2}{|c|}{ TOTAL } & 0.781 & 0.836 & 0.867 & 0.889 & 0.880 & \\
\hline \multicolumn{2}{|c|}{ REQUIREMENT } & 0.615 & 0.750 & 0.820 & 0.880 & 0.880 & \\
\hline
\end{tabular}

Assumes intrinsic extrapolated improvement of gold with increasing wavelength

\begin{tabular}{|l|}
\hline \multicolumn{1}{|c|}{ Coating specification } \\
\hline Contamination Control Plan \\
\hline flight environment degradation \\
\hline - \\
measured data \\
Particulates and NVR \\
based on contamination \\
control modeling anchored \\
to past experiences \\
Ice based on preliminary \\
analyses based on thermal \\
cool-down modeling and \\
anticipated water content \\
of materials at launch. \\
These analyses are being \\
refined. \\
Carbon is a conservative \\
estimate based on similar \\
data obtained from GoES \\
Micrometeoroid data \\
based on L2 environment \\
and hypervelocity testing \\
of mirror samples \\
MR-211/OBS-102 \\
34
\end{tabular}




\section{Particullate Coverage is Estilmated from Schedlulle}

- For each stage of I\&T the duration and location of activities is known

- Using an estimate (requirement) of allowed fallout the incremental increase in percent areal coverage (PAC) is determined

- The main effects of particulate coverage is in loss of transmission

- Wavelength independent

- The Al\&T schedule gives a prediction of the pre-launch particulate load on each mirror

- A redistribution analysis is also conducted to see where the particles are likely to be after launch

\section{PMSA PAC Contamination}






\section{PMSA NVR Contamination}

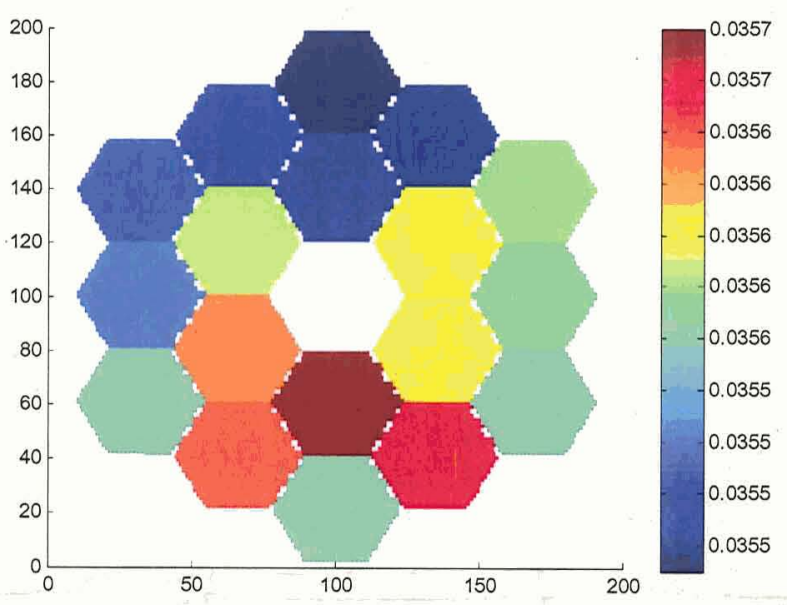

NVR $[\mu \mathrm{m}]$

\section{Dirrty Milirers}

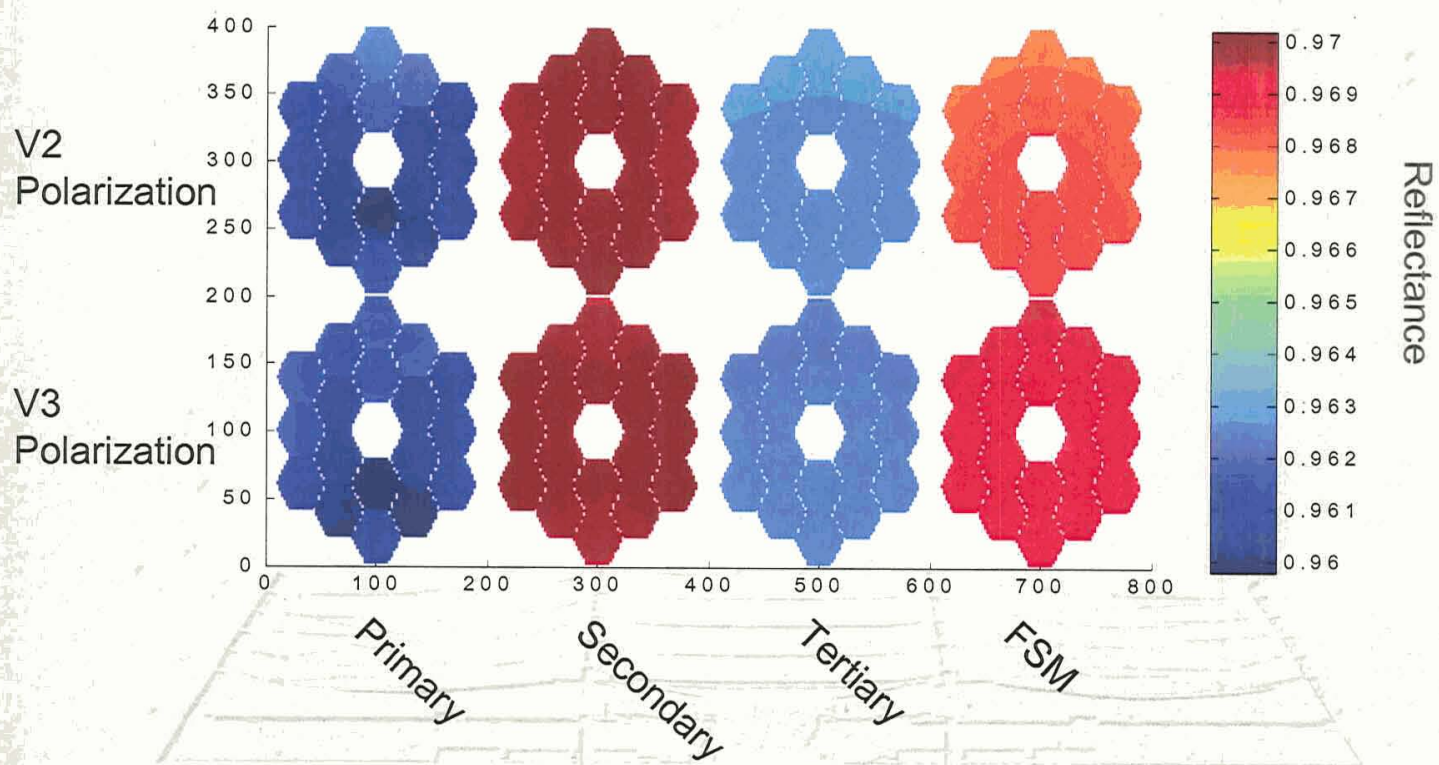




\section{Telescope Throughput}

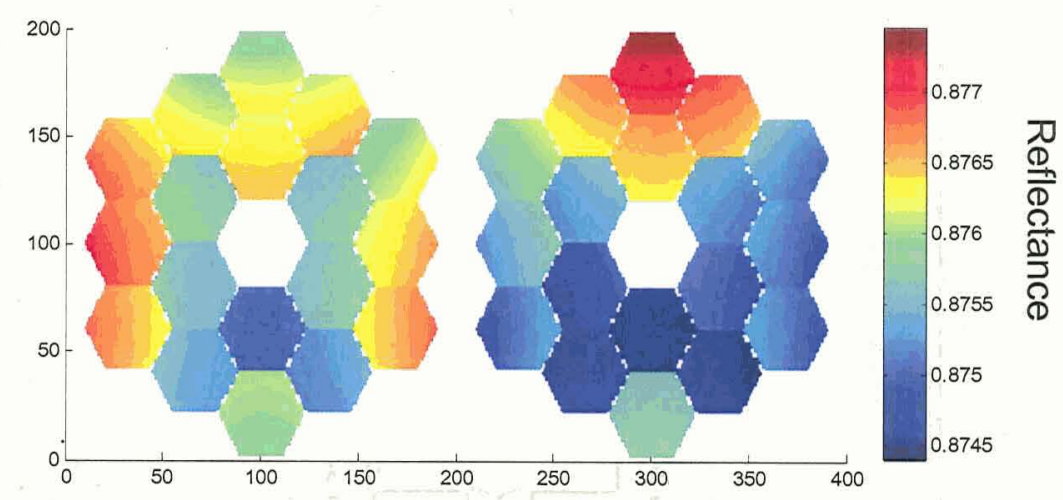

V2 Polarization V3 Polarization

\section{Integrated Tellescope Transmission Callgullation}

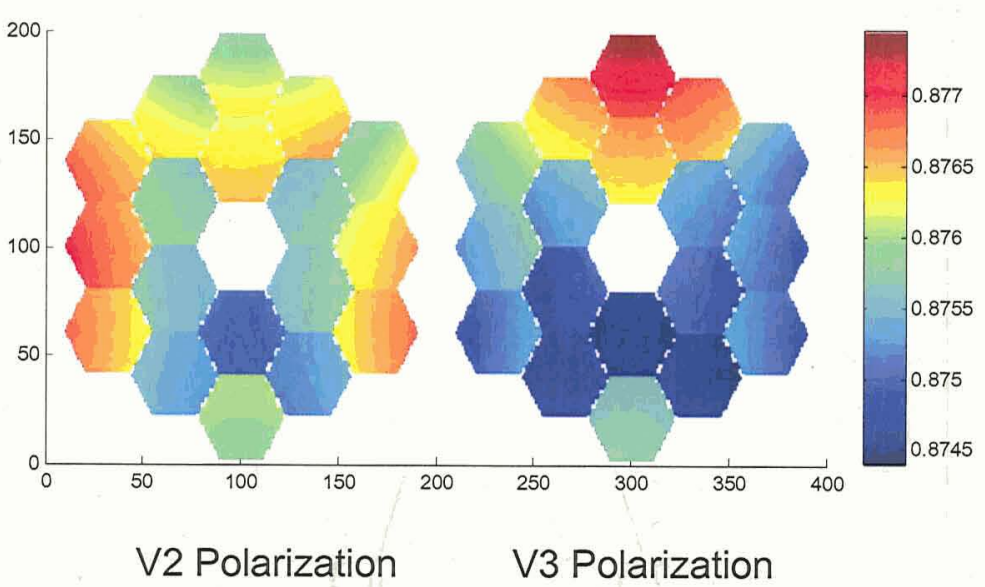

- Calculate the transmission for each mirror

- Reflectance using a contamination and ice modified stack

- Angle of incidence effects

- PAC for each mirror

- Both polarizations 


\section{Wavelength Sweep}

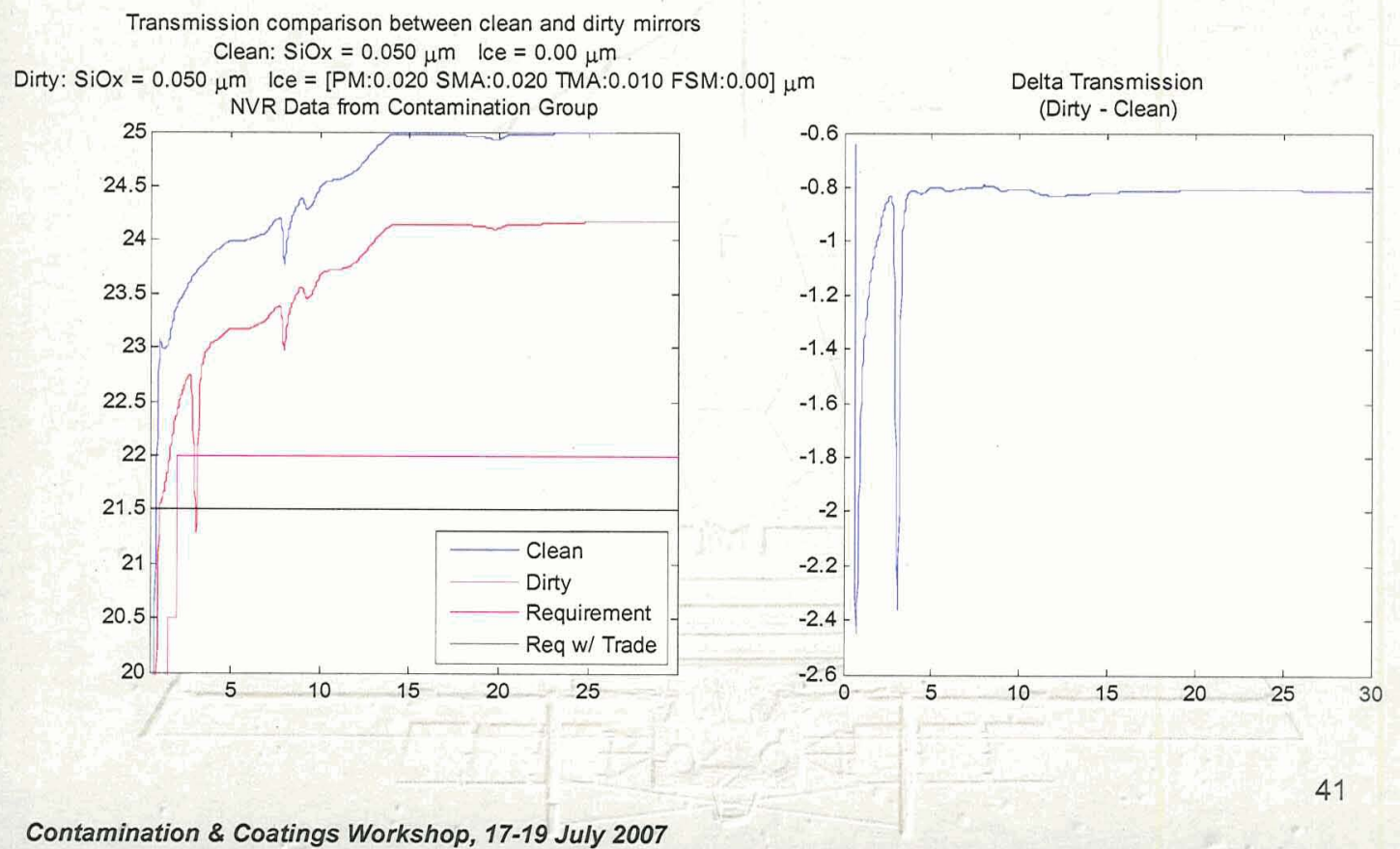

\section{Summary}

- JWST is a challenging mission in all respects

- Science performance is sensitive to contamination levels

- Mission demands open architecture

- Open architecture places greater emphasis on contamination requirements

- Contamination requirements established are directly derived from the Mission requirements 